This is the author's final, peer-reviewed manuscript as accepted for publication. The publisher-formatted version may be available through the publisher's web site or your institution's library.

\title{
Impact of a brief intervention on physical activity and social cognitive determinants among working mothers: a randomized trial
}

Emily L. Mailey • Edward McAuley

\section{How to cite this manuscript}

If you make reference to this version of the manuscript, use the following information:

Mailey, E. L., \& McAuley, E. (2014). Impact of a brief intervention on physical activity and social cognitive determinants among working mothers: A randomized trial.

Retrieved from http://krex.ksu.edu

\section{Published Version Information}

Citation: Mailey, E. L., \& McAuley, E. (2014). Impact of a brief intervention on physical activity and social cognitive determinants among working mothers: A randomized trial. Journal of Behavioral Medicine, 37(2), 343-355.

Copyright: @ Springer Science+Business Media New York 2013

Digital Object Identifier (DOI):10.1007/s10865-013-9492-y

Publisher's Link: http://link.springer.com/article/10.1007/s10865-013-9492-y

This item was retrieved from the K-State Research Exchange (K-REx), the institutional repository of Kansas State University. K-REx is available at http://krex.ksu.edu 
Impact of a brief intervention on physical activity and social cognitive determinants among working mothers: A randomized trial

\author{
Emily L. Mailey ${ }^{1 \mathrm{a}} \&$ Edward McAuley ${ }^{2}$
}

${ }^{1}$ Kansas State University
${ }^{2}$ University of Illinois at Urbana-Champaign

${ }^{a}$ Note: Research was completed at University of Illinois at Urbana-Champaign

Address all correspondence to:

Emily L. Mailey, PhD

Kansas State University

Department of Kinesiology

8D Natatorium

Manhattan, KS 66506

Phone: (785) 532-7287

Fax: (785) 532-6486

Email: emailey@ksu.edu 


\begin{abstract}
Working mothers exhibit high levels of inactivity, and theory-based interventions to bolster physical activity within this population are needed. This study examined the effectiveness of a brief social cognitive theory-based intervention designed to increase physical activity among working mothers. Participants $(N=141)$ were randomly assigned to an intervention only, intervention plus follow-up support, or waitlist control condition. The intervention consisted of two group-based workshop sessions designed to teach behavior modification strategies using social cognitive theory. Data were collected at baseline, immediately post-intervention, and 6month follow-up. Results showed intervention participants exhibited short-term increases in physical activity, which were partially maintained six months later. Improvements in physical activity were mediated by increases in self-regulation and self-efficacy. This study provides some support for the effectiveness of a brief intervention to increase physical activity among working mothers. Future programs should explore alternative support mechanisms which may lead to more effective maintenance of initial behavior changes.
\end{abstract}

Keywords working mothers; exercise adherence; social cognitive theory; physical activity; selfefficacy; goal setting 


\section{Introduction}

Although the physical and psychological health benefits of physical activity are wellestablished, more than half of U.S. adults do not participate in enough physical activity to realize these benefits (CDC, 2007). Working mothers are one segment of the population that exhibits particularly high levels of inactivity, which can largely be attributed to the numerous real and perceived physical activity barriers they encounter. Although over $70 \%$ of mothers now work (Bureau of Labor Statistics, 2009), an imbalance in the sharing of domestic responsibilities persists, and most women still bear the bulk of the responsibility for household and childcare duties (Bartley et al., 2005). As a result, they have limited access to personal leisure time and physical activity is neglected (Brown et al., 2001; Cramp \& Bray, 2011). The "role overload" experienced by many working mothers also contributes to elevated levels of stress, fatigue, depression, and anxiety in this population (Bekker et al., 2000; Floderus et al., 2008; Luecken et al., 1997). Physical activity has consistently been shown to alleviate these negative health outcomes, which could result in significant improvements in quality of life among working mothers (Long \& Haney, 1988; Urizar et al., 2005). Although increasing physical activity among working mothers is an important priority, attention to this population in the physical activity literature has been extremely limited to date.

Cross-sectional studies have consistently shown self-reported rates of physical activity participation among mothers to be significantly lower than those of women without children (Burke et al., 2004; Marcus et al., 1994; Verhoef \& Love, 1994). Furthermore, there is evidence that physical activity levels decline across the transition into motherhood, with several studies showing over $50 \%$ of mothers who were regularly active prior to motherhood were no longer 
meeting the national physical activity recommendations after having a child (Albright, Maddock, et al., 2005; McIntyre \& Rhodes, 2009). This problem appears to be exacerbated in working mothers; Project Viva found young mothers who worked 35 to 44 hours per week during pregnancy were more than three times as likely to be inactive as women who were not working, and women working more than 45 hours per week were over five times as likely to be inactive (Pereira et al., 2007). Specific exercise barriers identified by mothers include lack of time, lack of energy, lack of social support, lack of childcare, and guilt associated with taking time away from family obligations to participate in a "selfish" leisure activity such as physical activity (Albright, Maddock, et al., 2005; Brown et al., 2001; Cramp \& Bray, 2011; Miller \& Brown, 2005; Pereira et al., 2007; Verhoef \& Love, 1994).

One of the most widely used theoretical frameworks for understanding health behaviors, including physical activity, is Social Cognitive Theory (SCT). Bandura (2004) proposes a model in which self-efficacy interacts with outcome expectations, goals, and perceived environmental facilitators and impediments to influence behavior. Efficacy beliefs (i.e., confidence in one's capabilities to execute a specific course of action) are considered to be the primary impetus to action, with individuals being unlikely to engage in a behavior if they do not believe they are capable of producing the desired effects. Additionally, Bandura (1988) contends that selfregulation (i.e., guiding one's own actions by setting personal goals and planning courses of action to achieve them) is essential for maintaining a complex behavior such as physical activity. Expected positive outcomes provide the motivation and incentive to encourage self-regulatory behaviors. Finally, SCT asserts that behavior is not determined solely by individual factors. Individuals also encounter interpersonal and environmental factors that either facilitate the behavior or present barriers that must be overcome. 
SCT is an ideal theory for effecting changes in physical activity among working mothers for several reasons. First, its core constructs are modifiable and can be targeted in interventions. Second, working mothers who enroll in a physical activity intervention likely possess the motivation to change their behavior (a critical precondition for change identified by Bandura), but lack the self-efficacy and effective self-regulatory strategies to ensure physical activity is a priority. Well-designed SCT-based interventions provide individuals with the means to successfully regulate their behavior and develop confidence to adopt and maintain a regular physical activity program.

Interventions founded on SCT have often employed a classroom-based approach in order to provide individuals with the knowledge and skills they need to change their behavior. For example, participants will attend a series of classes and learn to set effective goals, identify realistic outcome expectations, and develop strategies for overcoming physical activity barriers. A small number of such interventions have specifically targeted mothers, and the results thus far have been promising, with all studies reporting some positive changes in behavioral outcomes (Cody \& Lee, 1999; Cramp \& Brawley, 2006; Fahrenwald et al., 2004; Fjeldsoe et al., 2010). However, to date, no intervention studies have specifically focused on working mothers, a segment of the population who faces unique challenges and could potentially reap considerable physical and mental health benefits from such a program. Importantly, working mothers are unlikely to be able to devote a large amount of time to learning behavioral strategies if they already feel they are too busy. Traditional classroom-based interventions that meet often and span across multiple months, therefore, would not likely be effective or feasible in this population. 
Thus, the purpose of the present study was to examine the effectiveness of a brief intervention (i.e., comprised of only two intensive sessions) for increasing physical activity among working mothers. We hypothesized that individuals who participated in the intervention would exhibit increases in physical activity compared to those assigned to a waitlist control condition. An important concern related to the brief intervention approach is whether participants will receive the tools and support needed to make lasting changes in their physical activity behavior. In previous interventions that have included follow-up assessments of key outcomes, a large percentage of participants have regressed to (or close to) baseline levels despite showing improvements immediately post-intervention (e.g., Calfas et al., 2000; Cody \& Lee, 1999). To this end, some continued contact with participants beyond the termination of the face-to-face intervention may be necessary. Telephone counseling has been demonstrated to be an effective means of ongoing support (Albright, Pruitt, et al., 2005); thus, a second purpose of this study was to explore the extent to which telephone support may enhance long-term maintenance of physical activity changes. We hypothesized that individuals who received telephone support would report higher levels of physical activity at 6-month follow-up than individuals who did not receive continuing support. Finally, this study was designed to examine social cognitive determinants of short- and long-term changes in physical activity. We hypothesized that individuals who participated in the intervention would exhibit increases in self-efficacy and self-regulation (i.e., goals and planning) compared to those in the control condition. Additionally, we predicted changes in self-efficacy and self-regulation would be significantly associated with physical activity behavior change among intervention participants, and that intervention effects on physical activity would be mediated by these variables.

\section{Methods}




\section{Participants}

Participants $(N=141)$ were females in central Illinois aged 25-52 who were employed at least 25 hours per week and had at least one child under age 15 living at home. Additional inclusion criteria included willingness to be randomized, ability to attend two workshop sessions, ability to access the internet, and completion of the Physical Activity Readiness Questionnaire (PAR-Q). Individuals who responded "yes" to one or more questions on the PAR-Q were required to obtain physician clearance before proceeding with participation. Women who were already meeting or exceeding the national physical activity recommendations (i.e., more than 150 minutes of moderate activity per week for the previous 2 months) based on self-reported activity levels were excluded.

Measures

\section{Demographics}

A demographics questionnaire was administered to ascertain participants' age, race, education, income, marital status, employment status, and parenthood status (including number and ages of children).

\section{Physical activity: Accelerometer}

Physical activity was measured objectively using Actigraph accelerometers (Manufacturing Technology Inc., Pensacola, FL). The accelerometer (Model GT3X) is a small $(3.8$ x 3.7 x 1.8 $\mathrm{cm})$, lightweight (27 $\mathrm{g}$ ) device that is worn on a belt over the non-dominant hip. Participants were asked to wear this device for all waking hours, except when showering or swimming, for seven consecutive days. In addition, participants were asked to record the times they started and 
stopped wearing the monitor each day on an accelerometer log. Data were downloaded and digitally converted to "activity counts" per minute (i.e., one epoch), and then cleaned and reduced using MeterPlus 4.2 software (Santech Health, San Diego, CA). Only days with at least ten hours of valid wear time were included in the analyses, and hours with greater than 30 minutes of consecutive zeros were considered invalid (i.e., non-wearing). Total activity counts were summed and averaged across the total number of valid days to get a total daily activity score. Additionally, established cutpoints (Freedson et al., 1998) were used to identify minutes of moderate/vigorous physical activity (>1952 counts per minute). Average daily moderate/vigorous activity was calculated by first summing the total valid moderate and hard activity counts, then dividing by the number of valid days.

\section{Leisure-time Physical Activity}

Self-reported physical activity was also assessed using the Godin Leisure-Time Exercise Questionnaire (Godin \& Shephard, 1985). This brief measure asks participants to report the current frequency of engaging in strenuous (e.g., running), moderate (e.g., easy bicycling or swimming), and light (e.g., bowling or golf) physical activity for at least 15 minutes per session during a typical week. A total weekly leisure activity score was calculated by multiplying the frequencies of strenuous, moderate, and light activities by nine, five, and three, respectively, and then summing the products. This questionnaire is widely used and has previously demonstrated adequate test-retest reliability and concurrent validity with objective measures of physical activity and energy expenditure (Jacobs, et al., 1993).

\section{Self-efficacy}


The Exercise Self-efficacy Scale (McAuley, 1993) and Barriers Self-Efficacy Scale (McAuley, 1992) were used to assess self-efficacy. The Exercise Self-efficacy Scale measures participants' beliefs in their ability to continue exercising five times per week in the future, at a moderate intensity, for 30 minutes per session. The Barriers Self-Efficacy Scale assesses participants' perceived capabilities to exercise regularly in the face of commonly identified barriers to participation (e.g., bad weather, schedule conflicts). For both measures, higher scores reflect greater self-efficacy (range: 0-100). Internal consistency was excellent for both Exercise Selfefficacy ( $a=.99)$ and Barriers Self-Efficacy $(a=.91-.94)$.

\section{Outcome Expectations}

The Multidimensional Outcome Expectations for Exercise Scale (Wojcicki et al., 2009) consists of three subscales: physical outcome expectations (e.g., "Exercise will increase my muscle strength"), social outcome expectations (e.g., "Exercise will provide companionship") and selfevaluative outcome expectations (e.g., "Exercise will give me a sense of personal accomplishment"). Higher scores reflect more positive outcome expectations. Internal consistency was acceptable for the physical ( $a=.80-.90)$, self-evaluative ( $a=.84-.92)$, and social subscales $(a=.76-.80)$.

\section{Self-regulation}

The Exercise Goal-Setting Scale and the Exercise Planning and Scheduling Scale (Rovniak et al., 2002) were used to assess self-regulatory behavior. The Exercise Goal-Setting Scale consists of ten items related to goal setting, self-monitoring, and problem solving (e.g., "I usually keep track of my progress in meeting my goals"). The Exercise Planning and Scheduling Scale includes ten 
items related to scheduling and planning exercise as part of one's daily routine (e.g., "I schedule my exercise at specific times each week."). For each scale, higher scores reflect greater use of self-regulatory strategies. Internal consistency for both Goal-Setting ( $a=.90-.93)$ and Planning/Scheduling ( $a=.80-.90)$ was good.

\section{Social support}

The Social Support for Exercise Scale (Sallis et al., 1987) is a 10-item scale assessing the degree to which friends and family demonstrate verbal (e.g., "Gave me helpful reminders to be active") and behavioral (e.g., "Helped plan activities around my activity routine") support for physical activity behaviors in the previous 3 months. Higher scores reflect greater support from family and friends. Both the family $(a=.88-.92)$ and friend ( $a=.86-.95)$ subscales demonstrated good internal consistency.

Procedures

All procedures were approved by a University Institutional Review Board. Participant recruitment began in March, 2011 and follow-up data collection was complete in January, 2012. Participants were recruited via local media outlets (e.g., university email lists, newspaper article, radio segment), local organizations frequented by mothers (e.g., day care centers, public libraries), and local community groups aimed at women, mothers, and/or working professionals. Recruitment materials targeted women who were not currently meeting the national physical activity recommendations but were motivated to become more active. Individuals who responded to the advertisements were contacted via telephone or email to provide a description of the study, determine eligibility, and extend an offer to participate in the study. Participants who met inclusion criteria and agreed to participate were mailed a packet that included an informed 
consent document, the accelerometer, accelerometer log, and instructions for wearing the accelerometer and completing the questionnaires online. All individuals signed and returned the informed consent document prior to being included in the study. Participants were instructed to wear the accelerometer for one week and complete the questionnaires during the same week. After wearing the accelerometer for seven days, they sent the accelerometer and log back to the investigator in a provided self-addressed, stamped envelope.

For an overview of the study design, refer to Figure 1. Following completion of all baseline measures, participants were stratified by number of children and age of youngest child, and a computerized data management system was used to randomly assign participants to one of three conditions: intervention only $(n=47)$, intervention plus follow-up support $(n=48)$, or waitlist control ( $n=46)$. Randomization was completed by an independent investigator who was not involved with data collection or intervention delivery. For all groups, follow-up data were collected in the same manner as baseline data immediately post-intervention (1 month), and 6 months after baseline. During the 6-month study duration, the control group's only contact with the researcher was for data collection purposes. They did not receive any specific instructions relative to physical activity (i.e., they were free to continue their regular routines and exercise if they wished). After all 6-month follow-up data had been collected, participants in the waitlist control group were invited to attend a 2-hour workshop that summarized the content received by the participants in the intervention groups.

\section{Intervention}

The intervention took place during the first month following randomization. The intervention consisted of two interactive group-based sessions, spaced three weeks apart, which 
taught participants behavior modification strategies based on social cognitive principles. The number of women attending each session ranged from 4 to 11, with most groups having 6 to 8 participants. Participants in the "intervention only" and "intervention plus" groups were scheduled to attend separate sessions to avoid contamination between groups, but the content each group received was identical. The first and second sessions lasted approximately 2 hours and 1.5 hours, respectively. The sessions were guided by the study investigator, but were interactive and incorporated small and large group discussions and problem-solving activities. Sessions were held on evenings and weekends to accommodate participants' schedules, and childcare was provided on-site for those who needed it.

Table 1 outlines the SCT-based content of the intervention. A variety of strategies were utilized to target each of the core SCT constructs during the sessions. For example, to enhance self-efficacy participants set small, manageable goals and then shared their "success stories" with the group. Each workshop session also featured several brief video clips of "model" working moms (i.e., regularly active) discussing physical activity benefits and barriers, and providing advice for other working moms just getting started. Outcome expectations were addressed by asking participants to identify ways in which being active would benefit them as moms. Participants learned a number of strategies for self-regulating their behavior, including using "SMART" goal setting principles (i.e., specific, measurable, action-oriented, realistic, and timebased; Bovend'Eerdt et al., 2009), completing activity logs to monitor their progress, and scheduling exercise sessions on their calendars. They also received a pedometer (Yamax Digiwalker Pedometer; Model SW-200) and an accompanying electronic log for tracking their daily steps to facilitate goal setting and self-monitoring. Finally, to address facilitators and impediments, participants identified their biggest physical activity barriers and devised strategies 
for overcoming them. Because leaving home to exercise at a gym is not a viable option for many working mothers, all participants received a 1-hour session with a personal trainer, who worked with them to devise an exercise program that could be completed at home. Important facilitators such as social support and activity prompts were also discussed. Each participant received a handbook containing information, worksheets, and resources that complemented the topics addressed during the workshop sessions.

During the follow-up period (i.e., between month 1 and month 6 testing), participants in the intervention only condition received no further contact from the research team. Participants assigned to the intervention plus follow-up support condition received monthly telephone support calls from a study investigator to monitor their progress following the structured intervention. These contacts were brief ( $\sim 3-8$ minutes) and designed to provide support, feedback, and active problem solving strategies which were individualized based on the individual's current activity level and goal adherence. The phone calls were semi-structured in that they focused on topics specified in an interview guide (e.g., barriers encountered or anticipated, pedometer use, goals for the following month) but were flexible to accommodate participants' individual needs. If a participant could not be reached after three attempts, the investigator contacted her by email and asked her to send an update on her progress during the past month.

Statistical Analysis

Given that no randomized controlled physical activity trials designed specifically for working mothers have been conducted, a conservative effect size $(d=.3)$ was selected for the a priori power analysis. The trial was powered to detect a 3 (Group) x 2 (Time) interaction (i.e., 
short-term effect) for the proposed multivariate analyses with $80 \%$ power, which yielded a total sample size of 111 participants (37 per group). To account for $20 \%$ attrition, we planned to recruit a total of 135 participants (45 per group).

We used the multiple imputation analysis option in SPSS v.20 to impute missing values at 1 and 6 months. Missing data ranged from 16.3\% (accelerometer) to 19.9\% (questionnaires) at 1 -month follow-up, and from $24.8 \%$ (questionnaires) to $25.5 \%$ (accelerometer) at 6 month follow-up. All analyses were repeated using the original data set with missing data and findings were not substantially different from those conducted with the imputed data. The imputed sample results are reported herein.

To determine whether the intervention only and intervention plus groups exhibited differential patterns of change for any of the physical activity or social cognitive variables, we first conducted a series of 2 (Group) x 3 (Time) analyses of variance (ANOVAs). These analyses revealed no significant differences between the intervention only and intervention plus groups. Thus, the two intervention groups were combined for comparison to the control group for all subsequent analyses.

To determine the short and long-term effects of the intervention on physical activity, a series of three 2 (Group) x 3 (Time) repeated measures ANOVAs were used to compare activity levels between those in the intervention and control groups. The three primary physical activity outcomes were composite Godin Leisure-Time Exercise Questionnaire score, average daily counts (accelerometer), and average daily minutes of moderate/vigorous activity (accelerometer). 
To explore the role of social cognitive determinants, a series of repeated measures MANOVAs were used to investigate patterns of change in SCT model variables between groups. Dependent variables were grouped as self-efficacy (Barriers Self-efficacy Scale and Exercise Self-efficacy Scale composite scores), outcome expectations (physical, social, and selfevaluative subscale scores), goals (Exercise Goal-Setting Scale and Exercise Planning and Scheduling Scale composite scores), and social support (friend and family participation subscales of the Social Support for Exercise Scale). Subsequently, standardized residual change scores (baseline to post-intervention and baseline to follow-up) were calculated for the three physical activity variables as well as each SCT outcome, and correlation analyses were conducted to examine the relationships between changes in physical activity and changes in social cognitive determinants within the intervention condition. Both short- and long-term changes were examined. Finally, to determine whether the intervention effects on physical activity were mediated by changes in SCT constructs, three regression equations were tested for all significant correlates of changes in physical activity. Specifically, physical activity change was regressed on treatment group assignment, the SCT change variables were regressed on treatment group assignment, and physical activity change was regressed on group assignment and the SCT change variables. Mediation was established when treatment group assignment was no longer a significant predictor of changes in physical activity when the SCT change variables were included in the model.

\section{Results}

Participant Characteristics and Retention 
For a detailed view of participant flow through the study, see Figure 2. A total of 224 individuals initially expressed interest in the study. Of these, 141 individuals provided complete baseline data and were randomized. Baseline characteristics of the sample are displayed by group in Table 2. On average, participants were 37.3 years old and had approximately 2 children (range: 1-7). There was considerable variation in number and age of their children, but on average, the youngest child was 4.75 years old (range: 2 months- 15 years). A majority of participants were white, married, and working full-time. As a whole, the sample was welleducated and relatively affluent. T-tests and nonparametric tests revealed no significant differences between groups on any of the demographic variables.

Attrition was similar across conditions (Figure 2). Of the 141 randomized participants, 119 participated in post-intervention data collection. Eight participants dropped out prior to attending a workshop session due to schedule conflicts. The remainder discontinued participation due to lack of interest $(n=6)$, family/personal issues $(n=7)$, and schedule conflicts $(n=1)$. An additional ten participants were lost at the six-month follow-up. Four could no longer be reached by telephone or email, three were no longer interested, and three reported family/personal issues that prevented continuing participation.

A series of t-tests were conducted to determine whether participants who completed the study differed from those who dropped out following randomization on any demographic or baseline measures. Analyses revealed participants who completed the study were more educated $(t(139)=2.93, p=.005)$ and had higher daily activity $(t(139)=2.23, p=.03)$ and moderate/vigorous activity $(t(139)=2.82, p=.006)$ at baseline based on the accelerometer. It is not clear, however, whether the significant differences in accelerometer counts were a function of lower activity 
levels or poorer compliance with instructions for wear among those who dropped out, as the dropouts also had significantly less valid hours of wear time $(t(139)=2.33, p=.03)$ than those who completed the study.

Intervention Effects on Physical Activity

Descriptive statistics and effect sizes for all physical activity variables are presented by group in Table 3. For self-reported leisure-time physical activity, there was a significant main effect for time $\left[F(2,138)=28.00, p<.001, \eta^{2}=0.29\right]$, and the interaction effect was also significant $\left[F(2,138)=3.41, p=.04, \eta^{2}=0.05\right]$. Intervention participants reported a significantly larger increase in physical activity than control participants immediately following the brief intervention. During the follow-up period, intervention participants reported a slight decrease in leisure-time physical activity, but their 6-month self-reported physical activity score was significantly higher than both their baseline score and the 6-month score of their control group counterparts. For total physical activity measured by the accelerometer, there was a significant quadratic time effect $\left[F(2,138)=9.36 p<.001, \eta^{2}=0.12\right]$ and a nonsignificant interaction effect $\left[F(2,138)=1.70, p=.19, \eta^{2}=0.02\right]$. For accelerometer-measured moderate/vigorous activity, results were quite similar, with a significant quadratic time effect $\left[F(2,138)=12.24, p<.001, \eta^{2}\right.$ $=0.15]$ and a nonsignificant interaction effect $\left[F(2,138)=1.72, p=.18, \eta^{2}=0.02\right]$. The quadratic effects were driven by the intervention group, in which accelerometer-derived physical activity scores increased across the initial intervention period, but regressed to baseline values at the 6month follow-up.

Intervention Effects on Social Cognitive Outcomes 
Descriptive statistics and effect sizes for all social cognitive variables are presented by group in Table 4. For self-efficacy, there was a significant effect for time $[F(4,136)=2.64$, $\left.p=.04, \eta^{2}=0.07\right]$ and a nonsignificant interaction effect $\left[F(4,136)=0.70, p=.59, \eta^{2}=0.02\right]$. Examination of the mean values showed both exercise and barriers self-efficacy declined across the 6-month period within the control group, and within the intervention group, a small increase in self-efficacy from baseline to post-intervention was followed by a slightly larger decrease from post-intervention to follow-up. For outcome expectations, there was a marginally significant quadratic main effect $\left[F(6,134)=1.93, p=.08, \eta^{2}=0.08\right]$ and a significant quadratic interaction effect $\left[F(6,134)=2.18, p=.05, \eta^{2}=0.09\right]$. In general, outcome expectations did not change within the intervention group. Within the control group, outcome expectations increased somewhat from post-intervention to follow-up after declining significantly from baseline to postintervention. For self-regulation, both the main effect $\left[F(4,136)=18.03, p<.001, \eta^{2}=0.35\right]$ and interaction effect $\left[F(4,136)=9.89, p<.001, \eta^{2}=0.23\right]$ were significant. Self-reported use of goal setting and planning/scheduling increased significantly among intervention participants, and use of these self-regulatory strategies remained significantly higher at follow-up compared to baseline, whereas goal setting and planning/scheduling remained unchanged within the control condition across the 6-month period. Finally, for social support, there was a significant effect for time $\left[F(4,136)=4.52, p=.002, \eta^{2}=0.12\right]$, and a nonsignificant interaction effect $[F(4,136)=$ 1.87, $\left.p=.12, \eta^{2}=0.05\right]$. Overall, social support increased across the intervention period and decreased during the follow-up period in both groups, but the magnitude of the changes varied by treatment group and type of support. Specifically, the short- and long-term increases in social support were larger in the intervention group than the control group for support from family, but not from friends. 
Relationship Between Changes in Physical Activity and Changes in Social Cognitive Outcomes

Correlation analyses were utilized to determine whether changes in social cognitive constructs were related to changes in physical activity within the intervention group. Results are displayed in Table 5. Changes in exercise self-efficacy, barriers self-efficacy, goals, and planning/scheduling all displayed positive, significant relationships with changes in physical activity at both post-intervention and follow-up.

Mediation analyses were conducted first with self-reported physical activity as the dependent variable. Treatment group assignment significantly affected long-term changes in physical activity $(\beta=.180, p=.03)$, goals $(\beta=.262, p=.002)$, and planning/scheduling $(\beta=$ $.278, p=.001)$, and its effects on exercise self-efficacy $(\beta=.156, p=.065)$ and barriers selfefficacy $(\beta=.141, p=.096)$ approached significance. When treatment group and all four SCT change variables were included in the model as independent variables, only changes in exercise self-efficacy $(\beta=.439, p<.001)$ and planning/scheduling $(\beta=.223, p=.046)$ emerged as significant independent predictors of changes in physical activity. Treatment group no longer accounted for changes in physical activity $(\beta=.056, p=.44)$, which suggests the effects of the intervention were mediated by changes in self-efficacy and self-regulation. The mediation analyses were repeated with accelerometer-measured physical activity as the dependent variable. The effect of treatment group on long-term changes in physical activity was not significant ( $\beta=$ $.060, p=.48)$, so no further analyses were conducted.

\section{Discussion}


This study examined the effects of a brief intervention on physical activity and social cognitive determinants among working mothers. The results add to a growing body of literature that suggests theory-based interventions can be used to produce short-term changes in physical activity behaviors among women with children (Cody \& Lee, 1999; Cramp \& Brawley, 2006; Fahrenwald et al., 2004; Fjeldsoe et al., 2010). In the present study, the strongest effects were observed for self-reported physical activity, and accelerometer-measured physical activity also increased to a lesser degree. Given the time constraints faced by working mothers, it is encouraging that participants exhibited modest increases in physical activity after only a brief, intensive intervention. The extent to which these initial changes were maintained, however, is unclear. When examining changes in self-reported leisure-time physical activity, results showed intervention participants, despite reporting slight declines in physical activity from postintervention to follow-up, were still significantly more active than at baseline after six months. Unfortunately, the same maintenance effects were not observed for the objective (i.e., accelerometer-based) physical activity outcomes. After six months, intervention participants had regressed back to baseline activity levels for both total activity and moderate/vigorous activity.

There are several potential reasons for the differential effects based on the physical activity measure used. First, intervention participants may have over-reported their physical activity at follow-up in order to provide socially desirable responses. They were aware that the intervention was designed to increase their physical activity levels, so they may have (consciously or subconsciously) provided responses that would support the study objectives. Alternatively, it is important to consider that the Godin Leisure-Time Exercise Questionnaire focuses only on leisure-time physical activity, whereas the accelerometer encompasses all physical activity accumulated across the course of one's day. Thus, it is possible that participants 
genuinely increased the amount of planned, structured activity in which they engaged without significantly changing their overall physical activity levels. This explanation would certainly be plausible if the increased activity was primarily non-aerobic in nature (e.g., resistance exercises taught by the personal trainer), or if planned exercise sessions primarily took place indoors. Follow-up data were collected between September and December, so daily activities captured by the accelerometers may have declined due to seasonal effects. Finally, although accelerometers do eliminate some of the biases associated with self-reported data, they may be more sensitive to temporary physical activity impediments (e.g., illness or travel during the assigned week), whereas the Godin Questionnaire instructs individuals to report their activity levels during a "typical week."

Nonetheless, regardless of measure, intervention participants did exhibit at least some decline in physical activity during the follow-up period. These results are consistent with previous studies that have shown similar declines (e.g., Calfas et al., 2000; Cody \& Lee, 1999), and underscore the difficulty of promoting long-term changes in a complex behavior such as physical activity. Although the brief intervention approach is appealing for this population, the long-term results suggest such minimal contact may not be sufficient to produce long-term changes in physical activity. The challenge for future interventionists will be to devise strategies for bolstering sustained intervention effectiveness without unduly burdening participants.

Unfortunately, the hypothesis that declines in physical activity during the follow-up period would be attenuated by telephone support was not supported. The patterns exhibited by the intervention plus group were not significantly different from those exhibited by the intervention only group for all physical activity variables. Although previous studies (e.g., Albright, Pruitt, et al., 2005; Fahrenwald, et al., 2004) have demonstrated phone calls to be an 
effective means of affecting physical activity behavior, there are several possible explanations for the lack of effect in the present study. First, it is possible that the "dose" of the support provided was not sufficient to have a significant impact on participants' behavior. Phone calls were only received monthly and were, on average, about five minutes in duration. Perhaps participants would have benefitted from more intensive contact, given the brief nature of the intervention. Future studies should also consider using an evidence-based approach such as motivational interviewing to deliver such calls, and using a script to ensure the content of the calls remains consistent with the theoretical framework. Another possible explanation is that participants would have preferred follow-up support from their peers or family members, as opposed to a member of the research team. Previous studies have identified family and/or partner support as a key facilitator of physical activity among working mothers (Albright, Maddock, et al., 2005; Brown et al., 2001; Miller et al., 2002). Many individuals also indicated that they would have liked to have more contact with the other participants following the brief intervention. Such support could be offered via the internet (e.g., through email or social networking sites), which would allow participants to access support when it is convenient for them.

Analyses of the social cognitive variables revealed the intervention had the strongest effects on goal setting and planning/scheduling. Intervention participants reported large increases in use of these self-regulatory strategies immediately following the intervention which were maintained across the follow-up period. Self-regulatory skills such as goal setting, scheduling physical activity into one's day, and monitoring progress were explicitly incorporated into the intervention content, so it is not surprising that the data revealed improvements in these constructs. It is encouraging, however, that participants were still utilizing these important skills 
six months later, and correlation analyses indicated increases in goal setting and planning/scheduling were associated with increases in physical activity among participants who received the intervention. These results are consistent with previous tests of the SCT model in which self-regulation has demonstrated the strongest relationship with physical activity (Rovniak et al., 2002; Anderson, et al., 2006). For working mothers, maintaining an active lifestyle is likely to necessitate advance planning to make physical activity a priority in one's schedule. Future interventions should continue to equip participants with a variety of strategies for successfully regulating their behavior.

Although mean-level changes in self-efficacy did not differ significantly between groups across the study period as hypothesized, correlation analyses still supported a moderately strong relationship between changes in self-efficacy and changes in physical activity within the intervention condition. In fact, long-term changes in exercise self-efficacy emerged as a key mediator of long-term changes in self-reported physical activity. It is important to note that decreases in self-efficacy over the course of an intervention have been observed in other studies and are likely a function of participants overestimating their capabilities at baseline (McAuley et al., 2011). In addition, the remarkably low self-efficacy ratings (25-50\%) among participants in the present study deserve mention. When considered in the context of other studies that have shown efficacy scores in the range of 70-90\% even in inactive samples that face a variety of barriers (e.g., sedentary older adults, individuals with multiple sclerosis or coronary heart disease), this statistic highlights the extent to which numerous barriers make regular exercise a significant challenge for working mothers, and underscores the need for interventions within this population (McAuley et al., 2011; Motl, et al., 2009; Woodgate, et al., 2005). The ongoing challenge for physical activity researchers will be to design interventions that target self-efficacy 
in such a way that improvements in confidence to adhere to a physical activity program are generated and maintained in a majority of participants. Interventions with working mothers should continue to focus on teaching self-regulatory strategies and promoting social support as means for reducing perceptions of barriers and ultimately enhancing self-efficacy and physical activity maintenance.

This study has a number of strengths. First, this was the first randomized controlled trial to focus specifically on working mothers, a segment of the population with an ongoing need for physical activity intervention. Thus it provides some preliminary data from which other intervention studies might be developed. Recent meta-analyses (e.g., Webb et al., 2010; Taylor et al., 2012) have shown health behavior interventions based on theory are likely to be more effective, and the content of the present intervention was grounded in Social Cognitive Theory. The study design allowed for the examination of both short- and long-term effects of the brief intervention, as well as the extent to which follow-up telephone support could enhance physical activity maintenance and the extent to which changes in physical activity were mediated by targeted theoretical constructs. To our knowledge this study had the largest sample size of physical activity interventions for mothers to date and was the first to use accelerometers to supplement self-reported physical activity measures.

The limitations of this study must also be acknowledged. The study sample was quite homogenous, particularly with regard to socioeconomic status. Nearly $90 \%$ of participants were college graduates, and half possessed an advanced degree. Additionally, annual household income exceeded $\$ 40,000$ in approximately $85 \%$ of the sample. Certainly further testing will be necessary to determine the extent to which the intervention content could be implemented 
effectively in less advantaged groups. On the other hand, the sample was quite diverse in that participants' children ranged in age from infants to teenagers, and due to participants' busy schedules, it was not possible to match participants with similar mothers to facilitate social interaction and modeling during the workshop sessions. It would be prudent to conduct additional analyses to determine whether the intervention effectiveness varied in subsets of the total sample. Finally, the use of a waitlist control group may be considered a limitation of the study design, as it does not allow one to rule out attention as a possible explanation for intervention effects. The analyses of social cognitive mediators, however, do suggest the intervention effects on self-reported physical activity were mediated by self-efficacy and selfregulation, which were associated with increases in physical activity in the intervention group only.

Future studies will need to consider alternative means of providing support following the brief intervention. Strategies to promote maintenance such as additional workshop sessions, websites that facilitate continued interaction among participants, or family-based interventions should be explored. Future studies might also consider ways in which the intervention could be modified for subsets of the working mother population (e.g., parents of very young children, single and low-income mothers), or consider incorporating exercise (e.g., additional personal training sessions, group exercise sessions) explicitly into the intervention. Developing interventions to promote physical activity among working mothers is an important public health priority, and this study provides a foundation which future studies can build upon to work towards the ultimate goal of developing an effective, sustainable program which can be disseminated to have broad impact on health and quality of life among working mothers. 


\section{Acknowledgements}

This study was supported by the Coca-Cola Company Doctoral Student Grant on Behavior Research Fund and the Raymond and Rosalee Weiss Research Endowment from the American College of Sports Medicine Foundation.

\section{References}

Albright, C. L., Maddock,, J. E., \& Nigg, C. R. (2005). Physical activity before pregnancy and following childbirth in a multiethnic sample of healthy women in Hawaii. Women \& Health, 42, 95-110.

Albright, C. L., Pruitt, L., Castro, C., Gonzalez, A., Woo, S., \& King, A. C. (2005). Modifying physical activity in a multiethnic sample of low-income women: One-year results from the IMPACT (Increasing Motivation for Physical ACTivity) project. Annals of Behavioral Medicine, 30, 191-200.

Anderson, E. S., Wojcik, J. R., Winett, R. A., \& Williams, D. M. (2006). Social-cognitive determinants of physical activity: The influence of social support, self-efficacy, outcome expectations, and self-regulation among participants in a church-based health promotion study. Health Psychology, 25, 510-520.

Bandura, A. (1988). Self-regulation of motivation and action through goal systems. In V. Hamilton, G. H. Bower, \& N. H. Frijda (Eds.), Cognitive perspectives on emotion and motivation (pp. 37-62). Kluwer Academic Publishers: Norwell, MA.

Bandura, A. (2004). Health promotion by social cognitive means. Health Education \& Behavior, 31, 143-164. 
Bartley, S. J., Blanton, P. W., \& Gilliard, J. L. (2005). Husbands and wives in dual-earner marriages: Decision-making, gender role attitudes, division of household labor, and equity. Marriage \& Family Review, 37, 69-94.

Bekker, M. H. J., de Jong, P. F., Zijlstra, F. R. H., \& van Landeghem, B. A. J. (2000).

Combining care and work: Health and stress effects in male and female academics. International Journal of Behavioral Medicine, 7, 28-43.

Bovend'Eerdt, T. J. H., Botell, R. E., \& Wade, D. T. (2009). Writing SMART rehabilitation goals and achieving goal attainment scaling: A practical guide. Clinical Rehabilitation, $23,352-361$.

Brown, P. R., Brown, W. J., Miller, Y. D., \& Hansen, V. (2001). Perceived constraints and social support for active leisure among mothers with young children. Leisure Sciences, 23, 131144.

Bureau of Labor Statistics. (2009). Labor force participation rate of mothers, 1975-2007. Available at http://www.bls.gov/opub/ted/2009/jan/wk1/art04.htm. Accessibility verified August 29, 2012.

Burke, V., Beilin, L. J., Dunbar, D., \& Kevan, M. (2004). Changes in health-related behaviours and cardiovascular risk factors in young adults: Associations with living with a partner. Preventive Medicine, 39, 722-730.

Calfas, K. J., Sallis, J. F., Nichols, J. F., Sarkin, J. A., Johnson, M. F., Caparosa, S., ... Alcaraz, J. E. (2000). Project GRAD: Two-year outcomes of a randomized controlled physical activity intervention among young adults. American Journal of Preventive Medicine, 18, $28-37$. 
Centers for Disease Control and Prevention. (2007). U.S. physical activity statistics. Available at http://apps.nccd.cdc.gov/PASurveillance/StateSumV.asp. Accessibility verified August 29, 2012.

Cody, R., \& Lee, C. (1999). Development and evaluation of a pilot program to promote exercise among mothers of preschool children. International Journal of Behavioral Medicine, 6 , 13-29.

Cramp, A. G., \& Brawley, L. R. (2006). Moms in motion: A group-mediated cognitive behavioral physical activity intervention. International Journal of Behavioral Nutrition and Physical Activity, 3, 23.

Cramp, A. G., \& Bray, S. R. (2011). Understanding exercise self-efficacy and barriers to leisuretime physical activity among postnatal women. Maternal and Child Health Journal, 15, $642-651$.

Fahrenwald, N. L., Atwood, J. R., Walker, S. N., Johnson, D. R., \& Berg, K. (2004). A randomized pilot test of "Moms on the Move": A physical activity intervention for WIC mothers. Annals of Behavioral Medicine, 27, 82-90.

Fjeldsoe, B. S., Miller, Y. D., \& Marshall, A. L. (2010). MobileMums: A randomized controlled trial of an SMS-based physical activity intervention. Annals of Behavioral Medicine, 39, $101-111$.

Floderus, B., Hagman, M., Aronsson, G., Marklund, S., \& Wikman, A. (2008). Self-reported health in mothers: The impact of age, and socioeconomic conditions. Women \& Health, 47, 63-86.

Freedson, P. S., Melanson, E., \& Sirard, J. (1998). Calibration of the Computer Science and Applications, Inc. accelerometer. Medicine \& Science in Sports \& Exercise, 30, 777-781. 
Godin, G., \& Shephard, R. J. (1985). A simple method to assess exercise behavior in the community. Canadian Journal of Applied Sport Sciences, 10, 141-146.

Jacobs, D. R., Ainsworth, B. E., Hartman, T. J., \& Leon, A. S. (1993). A simultaneous evaluation of 10 commonly used physical activity questionnaires. Medicine \& Science in Sports \& Exercise, 25, 81-91.

Kay, T. (1998). Having it all or doing it all? The construction of women's lifestyles in timecrunched households. Society and Leisure, 21, 435-54.

Long, B. C., \& Haney, C. J. (1988). Long-term follow-up of stressed working women: A comparison of aerobic exercise and progressive relaxation. Journal of Sport \& Exercise Psychology, 10, 461-470.

Luecken, L. J., Suarez, E. C., Kuhn, C. M., Barefoot, J. C., Blumenthal, J. A., Siegler, I. C., \& Williams, R. B. (1997). Stress in employed women: Impact of marital status and children at home on neurohormone output and home strain. Psychosomatic Medicine, 59, 352359.

Marcus, B. H., Pinto, B. M., Simkin, L. R., Audrain, J. E., \& Taylor, E. R. (1994). Application of theoretical models to exercise behavior among employed women. American Journal of Health Promotion, 9, 49-55.

McAuley, E. (1992). The role of efficacy cognitions in the prediction of exercise behavior in middle-aged adults. Journal of Behavioral Medicine, 15(1), 65-88.

McAuley, E. (1993). Self-efficacy and the maintenance of exercise participation in older adults. Journal of Behavioral Medicine, 16(1), 103-113.

McAuley, E., Mailey, E. L., Mullen, S. P., Szabo, S. N., Wójcicki, T. R., White, S. M., ... 
Kramer, A. F. (2011). Growth trajectories of exercise self-efficacy in older adults: Influence of measures and initial status. Health Psychology, 30, 75-83.

McIntyre, C. A., \& Rhodes, R. E. (2009). Correlates of leisure-time physical activity during transitions to motherhood. Women \& Health, 49, 66-83.

Miller, Y. D., \& Brown, W. J. (2005). Determinants of active leisure for women with young children - An "ethic of care" prevails. Leisure Sciences, 27, 405-420.

Miller, Y. D., Trost, S. G., \& Brown, W. J. (2002). Mediators of physical activity behavior change among women with young children. American Journal of Preventive Medicine, $23,98-103$.

Motl, R. W., McAuley, E., Snook, E. M., \& Gliottoni, R. C. (2009). Physical activity and quality of life in multiple sclerosis: Intermediary roles of disability, fatigue, mood, pain, selfefficacy, and social support. Psychology, Health, \& Medicine, 14, 111-124.

Pereira, M. A., Rifas-Shiman, S. L., Kleinman, K. P., Rich-Edwards, J. W., Peterson, K. E., \& Gillman, M. W. (2007). Predictors of change in physical activity during and after pregnancy: Project Viva. American Journal of Preventive Medicine, 32, 312-319.

Rovniak, L. S., Anderson, E. S., Winett, R. A., \& Stephens, R. S. (2002). Social cognitive determinants of physical activity in young adults: A prospective structural equation analysis. Annals of Behavioral Medicine, 24, 149-156.

Sallis, J. F., Grossman, R. M., Pinski, R. B., Patterson, T. L., \& Nader, P. R. (1987). The development of scales to measure social support for diet and exercise behaviors. Preventive Medicine, 16, 825-836. 
Taylor, N., Conner, M., \& Lawton, R. (2012). The impact of theory on the effectiveness of worksite physical activity interventions: A meta-analysis and meta-regression. Health Psychology Review, 6, 33-73.

Urizar, G. G., Hurtz, S. Q., Albright, C. L., Ahn, D. K., Atienza, A. A., \& King, A. C. (2005). Influence of maternal stress on successful participation in a physical activity intervention: The IMPACT project. Women \& Health, 42, 63-82.

Verhoef, M. J., \& Love, E. J. (1994). Women and exercise participation: The mixed blessings of motherhood. Health Care for Women International, 15, 297-306.

Webb, T. L., Joseph, J., Yardley, L, \& Michie, S. (2010). Using the internet to promote health behavior change: A systematic review and meta-analysis of the impact of theoretical basis, use of behavior change techniques, and mode of delivery on efficacy. Journal of Medical Internet Research, 12, e4.

Wojcicki, T. R., White, S. M., \& McAuley, E. (2009). Assessing outcome expectations in older adults: The multidimensional outcome expectations for exercise scale. Journal of Gerontology: Psychological Sciences, 64B, 33-40.

Woodgate, J., Brawley, L. R., \& Weston, Z. J. (2005). Maintenance cardiac rehabilitation exercise adherence: Effects of task and self-regulatory self-efficacy. Journal of Applied Social Psychology, 35, 183-197. 
Table 1. Social Cognitive Theory-based content of the intervention

\begin{tabular}{|c|c|}
\hline SCT Construct & Sample intervention activities \\
\hline Self-efficacy & $\begin{array}{l}\text {-Broaden definition of "exercise" to include small, manageable bouts } \\
\text {-Set realistic short-term goals to enjoy regular success } \\
\text {-Share positive accomplishments related to physical activity } \\
\text {-View video vignettes of active working moms }\end{array}$ \\
\hline $\begin{array}{l}\text { Outcome } \\
\text { expectations }\end{array}$ & $\begin{array}{l}\text {-Identify personally relevant benefits of physical activity } \\
\text {-Discuss realistic expectations for weight loss }\end{array}$ \\
\hline $\begin{array}{l}\text { Goals/ } \\
\text { self-regulation }\end{array}$ & $\begin{array}{l}\text {-Set SMART goals } \\
\text {-Schedule exercise sessions on calendar } \\
\text {-Complete weekly activity log } \\
\text {-Use pedometer as objective indicator of progress } \\
\text {-Outline long-term goals using behavior contract }\end{array}$ \\
\hline $\begin{array}{l}\text { Facilitators/ } \\
\text { impediments }\end{array}$ & $\begin{array}{l}\text {-Identify key barriers and develop strategies for overcoming them } \\
\text {-Meet with personal trainer to learn exercises that can be completed at home } \\
\text {-Complete social support contract } \\
\text {-Choose appealing rewards for meeting goals } \\
\text {-Use cues to action to prompt physical activity }\end{array}$ \\
\hline
\end{tabular}


Table 2. Participant demographics at baseline

\begin{tabular}{lccc}
\hline \multicolumn{1}{l}{ Variable } & Intervention only & $\begin{array}{c}\text { Mean (SD)/Freq (\%) } \\
\text { Intervention plus }\end{array}$ & Control \\
\hline Age & $38.33(7.13)$ & $37.15(6.72)$ & $36.35(6.06)$ \\
Number of children & $1.98(1.14)$ & $1.74(0.77)$ & $2.04(1.03)$ \\
Age of youngest child & $5.62(3.96)$ & $4.38(4.01)$ & $4.22(3.49)$ \\
Employment status & & & \\
$\quad$ Full-time & $43(89.6 \%)$ & $44(93.6 \%)$ & $38(82.6 \%)$ \\
$\quad$ Part-time & $5(10.4 \%)$ & $3(6.4 \%)$ & $8(17.4 \%)$ \\
Hours worked per week & $40.71(7.07)$ & $39.76(6.56)$ & $39.09(6.75)$ \\
Marital Status & $37(77.1 \%)$ & $40(85.1 \%)$ & $42(91.3 \%)$ \\
$\quad$ Married & $6(12.5 \%)$ & $4(8.5 \%)$ & $2(4.3 \%)$ \\
$\quad$ Divorced/separated & $2(4.2 \%)$ & $2(4.3 \%)$ & $1(2.2 \%)$ \\
$\quad$ Partnered/significant other & $3(6.3 \%)$ & $1(2.1 \%)$ & $1(2.2 \%)$ \\
$\quad$ Single & & & \\
Race & $38(79.2 \%)$ & $37(78.7 \%)$ & $38(82.6 \%)$ \\
$\quad$ White & $4(8.3 \%)$ & $5(10.6 \%)$ & $4(8.7 \%)$ \\
$\quad$ African American & $5(10.4 \%)$ & $4(8.5 \%)$ & $1(2.2 \%)$ \\
$\quad$ Asian & 0 & 0 & $2(4.4 \%)$ \\
$\quad$ Other & $1(2.1 \%)$ & $1(2.1 \%)$ & $1(2.2 \%)$ \\
$\quad$ Not disclosed & $8(16.7 \%)$ & $4(8.5 \%)$ & $6(13.1 \%)$ \\
Education & $13(27.1 \%)$ & $23(48.9 \%)$ & $16(34.8 \%)$ \\
$\quad$ CCollege Graduate & $27(56.2 \%)$ & $20(42.5 \%)$ & $24(52.2 \%)$ \\
$\quad$ College Graduate & $7(14.7 \%)$ & $7(14.9 \%)$ & $5(10.8 \%)$ \\
Advance Degree & $40(83.3 \%)$ & $38(80.9 \%)$ & $41(89.1 \%)$ \\
Annual Household Income & $1(2.1 \%)$ & $2(4.3 \%)$ & 0 \\
$\quad$ <40,000 & & & \\
$\quad$ \$40,000 & & & \\
Not disclosed & & &
\end{tabular}


Table 3. Intervention effects on physical activity

\begin{tabular}{lcccccc}
\hline & 1. Baseline & 2. Post-intervention & 3. Follow-up & $d$ & $d$ \\
& & $M(S D)$ & $M(S D)$ & $M(S D)$ & $(1-2)$ & $(1-3)$ \\
\hline GLTEQ & Intervention & $19.01(17.1)$ & $35.51(18.5)$ & $33.75(22.5)$ & .93 & .74 \\
& Control & $16.92(19.3)$ & $24.44(16.2)$ & $25.31(18.3)$ & .42 & .45 \\
MVPA & Intervention & $22.45(13.3)$ & $29.58(20.3)$ & $21.28(13.2)$ & .42 & -.09 \\
& Control & $20.87(13.6)$ & $21.08(14.9)$ & $19.42(11.7)$ & .01 & -.11 \\
& & & & & & \\
Total Counts & Intervention & $217591(74418)$ & $254682(94041)$ & $223190(71858)$ & .44 & .08 \\
& Control & $222057(64031)$ & $232553(75914)$ & $217239(57253)$ & .15 & -.08 \\
& & & & & & \\
\hline
\end{tabular}

Note. GLTEQ=Godin Leisure Time Exercise Questionnaire; MVPA=Moderate/Vigorous Physical Activity (accelerometer); Total Counts (accelerometer); 1-2=Effect size (Cohen's d) for change from baseline to post-intervention; 1-3=Effect size (Cohen's d) for change from baseline to follow-up 
Table 4. Intervention effects on social cognitive variables

\begin{tabular}{|c|c|c|c|c|c|c|}
\hline & & $\begin{array}{l}\text { 1. Baseline } \\
M(S D)\end{array}$ & $\begin{array}{l}\text { 2. Post-intervention } \\
M(S D)\end{array}$ & $\begin{array}{l}\text { 3. Follow-up } \\
M(S D)\end{array}$ & $\begin{array}{c}d \\
(1-2)\end{array}$ & $\begin{array}{c}d \\
(1-3)\end{array}$ \\
\hline \multirow[t]{2}{*}{ BARSE } & Intervention & $45.3(18.0)$ & $48.1(19.0)$ & $41.8(18.4)$ & .15 & -.19 \\
\hline & Control & $40.6(20.3)$ & 37.4 (22.5) & $34.5(23.0)$ & -.15 & -.28 \\
\hline \multirow[t]{2}{*}{ EXSE } & Intervention & $44.8(30.7)$ & $45.4(29.2)$ & $40.4(28.2)$ & .02 & -.15 \\
\hline & Control & 39.4 (31.6) & $32.5(27.0)$ & $30.0(26.1)$ & -.23 & -.32 \\
\hline \multirow[t]{2}{*}{ Physical OE } & Intervention & $28.0(2.47)$ & $27.8(2.05)$ & $27.9(2.10)$ & -.09 & -.04 \\
\hline & Control & $28.2(1.83)$ & $26.8(3.81)$ & $27.4(1.98)$ & -.47 & -.42 \\
\hline \multirow[t]{2}{*}{ Self-eval OE } & Intervention & $22.3(2.54)$ & $22.4(2.06)$ & $22.5(1.92)$ & .04 & .09 \\
\hline & Control & $22.5(2.35)$ & $20.9(3.58)$ & $21.6(2.11)$ & -.53 & -.40 \\
\hline \multirow[t]{2}{*}{ Social OE } & Intervention & $12.0(3.01)$ & $12.1(2.51)$ & $12.4(2.63)$ & .04 & .14 \\
\hline & Control & $12.5(2.97)$ & $11.2(2.83)$ & $11.9(2.83)$ & -.45 & -.21 \\
\hline \multirow[t]{2}{*}{ Goals } & Intervention & $19.5(7.86)$ & $28.3(7.63)$ & $25.0(8.61)$ & 1.14 & .67 \\
\hline & Control & $20.6(9.52)$ & $20.2(8.90)$ & $20.8(9.37)$ & -.04 & .02 \\
\hline \multirow[t]{2}{*}{ Planning } & Intervention & $16.1(5.63)$ & $24.5(7.69)$ & $22.1(7.79)$ & 1.25 & .88 \\
\hline & Control & $16.0(5.89)$ & $18.4(7.12)$ & $17.6(7.06)$ & .37 & .25 \\
\hline \multirow[t]{2}{*}{ Family support } & Intervention & $22.3(7.84)$ & $26.5(8.56)$ & $23.9(8.31)$ & .51 & .20 \\
\hline & Control & $22.1(7.25)$ & $22.7(8.11)$ & $20.4(6.71)$ & .08 & -.24 \\
\hline \multirow[t]{2}{*}{ Friend support } & Intervention & $17.9(6.94)$ & $21.3(9.15)$ & 20.4 (7.66) & .42 & .34 \\
\hline & Control & $19.5(8.67)$ & $20.8(10.0)$ & $20.0(7.80)$ & .14 & .06 \\
\hline
\end{tabular}

Note. BARSE=Barriers Self-Efficacy; EXSE=Exercise Self-Efficacy; OE=Outcome

Expectations; 1-2=Effect size (Cohen's d) for change from baseline to post-intervention; 1-

$3=$ Effect size (Cohen's d) for change from baseline to follow-up 
Table 5. Correlations among residual change in physical activity and social cognitive determinants (intervention participants only)

\begin{tabular}{|c|c|c|c|c|c|c|}
\hline & & $\mathrm{m} 0-\mathrm{m} 1$ & & & $\mathrm{~m} 0-\mathrm{m} 6$ & \\
\hline & GLTEQ & Counts & MVPA & GLTEQ & Counts & MVPA \\
\hline EXSE & $.38 * *$ & $.34 * *$ & $.37 * *$ & $.60 * *$ & $.27 * *$ & $.30 * *$ \\
\hline BARSE & $.22 *$ & $.32 * *$ & $.38 * *$ & $.46^{* *}$ & $.24 *$ & $.25 *$ \\
\hline Physical OE & .01 & .09 & .09 & .19 & .08 & .02 \\
\hline Self-eval OE & .02 & .07 & .09 & .13 & .10 & .04 \\
\hline Social OE & .00 & -.06 & -.02 & -.02 & -.16 & -.15 \\
\hline Goals & $.30 * *$ & $.32 * *$ & $.35 * *$ & $.36 * *$ & $.31 * *$ & $.27 * *$ \\
\hline Planning & $.45 * *$ & $.42 * *$ & $.45^{* *}$ & $.45^{* *}$ & $.36 * *$ & $.35 * *$ \\
\hline Family support & .06 & -.08 & -.03 & $.28 * *$ & -.07 & .01 \\
\hline Friend support & $.21 *$ & -.01 & .05 & .10 & .00 & -.08 \\
\hline
\end{tabular}

Note. GLTEQ=Godin Leisure Time Exercise Questionnaire; MVPA=Moderate/Vigorous Physical Activity (accelerometer); Counts (accelerometer); BARSE=Barriers SelfEfficacy; EXSE=Exercise Self-Efficacy; OE=Outcome Expectations $* p<.05 * * p<.01$ 
Figure 1. Overview of study design

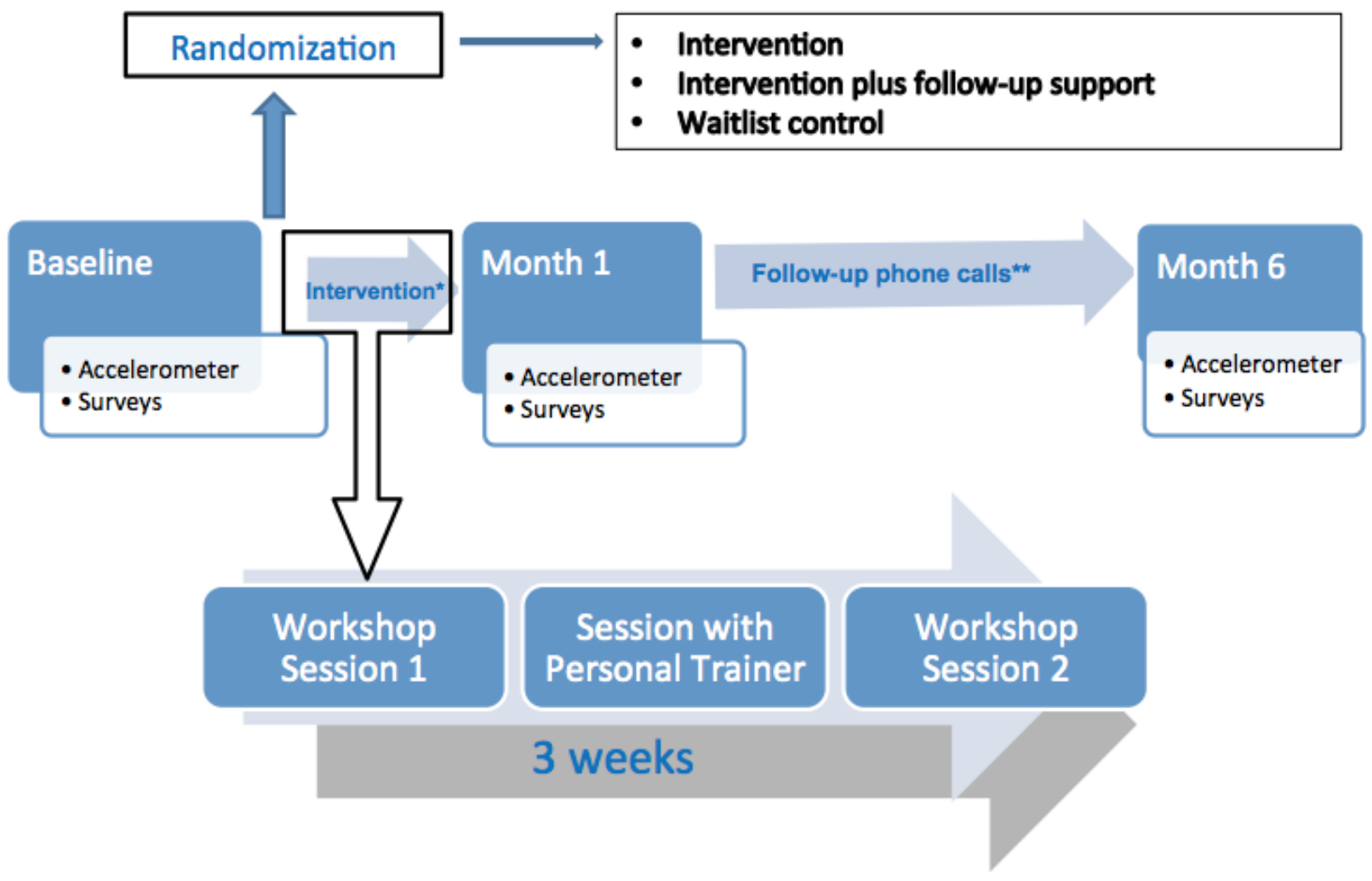

*Received by Intervention and Intervention plus follow-up support groups

**Received by Intervention plus follow-up support group only 
Figure 2. Participant flow through the study

\section{CONSORT Diagram}

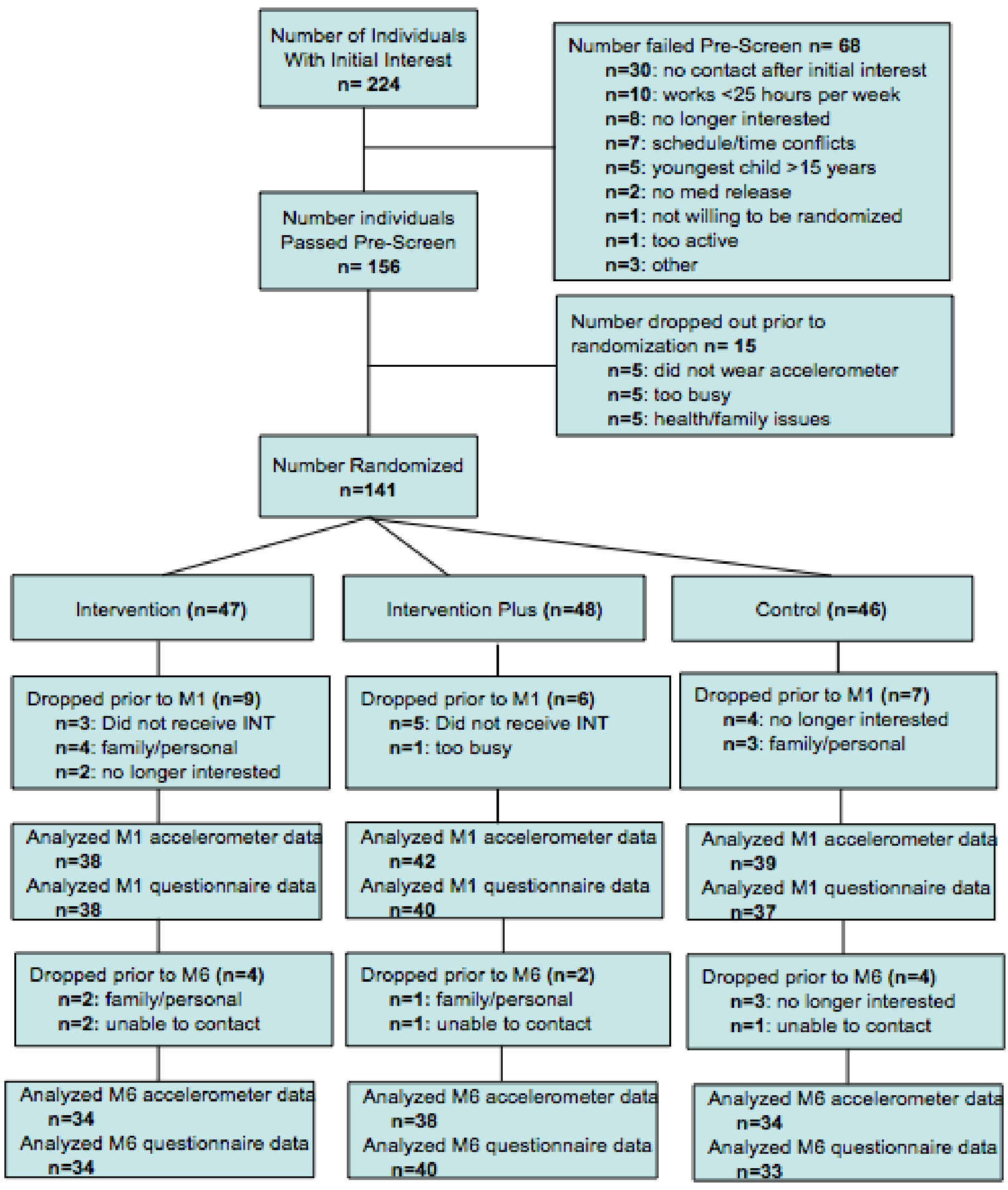

